\title{
Research on Effective and Intelligent Resource Management in Internet Computing
}

\author{
FuFang $\mathrm{Li}^{1}$, Dongqing Xie ${ }^{1}$, Deyu $Q i^{2}$, Guowen Xie ${ }^{3}$, Wenbin Chen ${ }^{1}$ and Lingxi Peng ${ }^{1, *}$ \\ ${ }^{1}$ School of Computer Science and Educational Software, GuangZhou University, Guangzhou 510006, P. R. China \\ ${ }^{2}$ School of Computer Science and Engineering, South China University of Technology, Guangzhou 510006, P. R. China \\ ${ }^{3}$ School of Life Science, GuangZhou University, Guangzhou 510006, P. R. China
}

Received: 24 Mar. 2013, Revised: 25 Jul. 2013, Accepted: 27 Jul. 2013

Published online: 1 Mar. 2014

\begin{abstract}
Internet Computing, such as web computing, grid computing and cloud computing, has been a research focus in recent years. Resource management and scheduling is one of the most basic and important problem in this area, and is far to be resolved satisfactorily. This paper presents a novel effective and intelligent Resource Management Model (and Algorithm) for Internet Computing based on Agents, Small-world network theory and Distributed hash table (namely RMMIC_ASD and RMAIC_ASD). Adopting the thoughts of agents, small-world network theory and distributed hash table, the intelligence, self-adaptability, robustness, performance and efficiency of internet resource management and scheduling can be greatly improved. Simulation experiments show that the proposed model and algorithm are feasible and rational and works better than similar algorithms.
\end{abstract}

Keywords: Internet Computing, Resource Management, Agents, Small-world Network Theory (SNT), Distributed Hash Table (DHT)

\section{Introduction and Related Work}

From web computing, grid computing to newly-minted cloud computing, internet-based distributed computing (i.e. Internet Computing) has been a hot spot in recent years. And more, Internet Computing has also been treated as a core scientific strategy by the academia, industry and even nations all over the world. On account of the character of distribution, heterogeneity, autonomy and dynamically changing of Internet Computing environment, resource management and scheduling is a key and challenging problem which should be resolved firstly.

To solve the problem of Internet Computing resource management, many researchers had done much fruitful work. Eric [1] developed a correlated resource model of Internet end hosts based on real-trace data taken from several volunteer computing projects, including SETI@ home. Their resource model is based on statistical analysis of host computational power, memory, and storage as well as how these resources change over time and the correlations among them. They validated and showed the utility of the model by applying it to a resource allocation problem for Internet-distributed applications. Compared with naive models and Grid resource models, their model is up to 130 percent more accurate. In paper [2], to enforce dynamic SLAs and to improve services offered to applications, authors proposed a new monitoring method to control the resource utilization and to reconfigure them when necessary in order to match analytical expressions used to describe the service parameters. The proposed method called flatness based trajectory tracking, considers time delay transfer by using delay predictor and provides value-added services for grid applications over Internet. Moreover, discrete-time simulation under GridSim simulator illustrates its feasibility and efficiency to provide guarantees for critical grid traffics with timely execution requirements and to match expressive data-transfer SLAs. A fast resource provisioning mechanism for internet services was developed for cloud applications by restoring previously created VM snapshots of fully initialized application in paper [3]. They proposed a set of optimizations, including working set estimation, demand prediction, and free page avoidance, that allow an application to start running with only partially loaded memory, yet without noticeable performance penalty during its sub-sequent execution.

\footnotetext{
*Corresponding author e-mail: harvardgd@ 163.com
} 
Experimental results indicate that Twinkle can provision VMs and restore the QoS significantly faster than the current approaches. Based on analyzing the characteristics of dynamics Internet resources, Peng [4] presented a computational model of the dynamic Internet resource allocation and some related distributed algorithms. Experimental results show that the models and the algorithms are effective and the ratio of the rejected request can be decreased drastically. Paper [5] constructs a scalable distributed resource information service (SDIRIS) infrastructure, and a related efficient multiple-attribute range query algorithm is presented. Their proposed infrastructure of SDIRIS can realize resource information publication and query efficiently. By using several run-time statistics of both workloads and system as indicators of busty and load condition, Liu [6] present a decentralized resource allocation approach for Decentralized Response-time Guarantees in storage system. Their approach can provide response time guarantees for multiple concurrent workloads sharing a back-end storage system in a distributed manner without assuming any support from the storage itself. Demonstrations show that their approach can simultaneously meet the response-time requirements effectively.

Although the above studies have obtained a lot of achievements, due to the complex and dynamically changing Internet Computing environment, the efficiency and real-time character of Internet resource management and scheduling is still far to be resolved satisfactorily. Agent technology has provided a new way in solving the problem of Internet Computing resource management and task scheduling which needs more cooperation, and has been widely used in many area[7,8,9,10]. Manish [10] presents a mechanism for Active Resource Management (ARM) in a differentiated services environment based on static provisioning of resources. To address the problem of either leading to wasted bandwidth or leaving applications resource-starved in static reservation approach, ARM dynamically reallocate resources based on current network state and applications requirements. The experimental evaluation presented shows that ARM can obtain sound efficiency in resource management. Recent research has proved that many real networks such as WWW and Internet possess typical character of SNT (Small-world Network Theory) [11,12]. Organized according to the theory of SNT, resources in Internet Computing environment are more reachable to each other, which would largely accelerate the searching speed while scheduling the resources. Distributed Hash Table (DHT) has been widely used in distributed resource organization, management, discover and so on $[13,14]$. And more, DHT has also become one of the most important infrastructures for distributed large-scale application system.

This paper tries to present a novel effective and intelligent Resource Management Model for Internet Computing environment based on Agents, SNT and DHT
(RMMIC_ASD). In the model of RMMIC_ASD, we propose a unified description approach to describe Internet resources and resource request from the users. In order to obtain good efficiency and performance, we conduct Small-world Network Theory, Agents technology and Distributed Hash Table algorithm to construct the model. We also give out a novel Resource Management Algorithm for Internet Computing based on Agents, SNT and DHT (RMAIC_ASD) related to the model of RMMIC_ASD. Experiments show that higher efficiency and performance can be obtained compared with similar approaches.

The rest of the paper is organized as follows: in Section 2, we discuss the presented model of RMMIC_ASD. We then discuss the effective resources management and scheduling algorithm (namely RMAIC_ASD) complying with the proposed model in Section 3. To evaluate the efficiency and performance of our model and related algorithm, we introduce the experimental results and analyses in section 4. Finally, we summarize our research work in Section 5.

\section{Effective and Intelligent Resource Management Model of RMMIC RASD $_{-}$}

In this section we firstly introduce the description approach of Internet Computing resources. Then we present the resource management model of RMMIC_ASD in detail.

\subsection{Description Model of Internet Computing Resources}

Its widely accepted that service-based virtual computing has become the development trend of Internet Computing. In complex dynamically changing and heterogeneous Internet Computing environment, resource requests addressed by users are put forward in the form of virtual service requests. Correspondingly, various heterogeneous Internet resources are provided in the form of virtual services in Internet Computing system. Hence, we can describe various Internet resources in a unified form of service. In order to organize and manage Internet resources effectively, this paper conducts Resource Vector (RV) as the unified description form for all kinds of Internet resources, i.e.

$$
R V=\left\{R A t t_{1}, R A t t_{2}, \ldots \ldots, R A t t_{n}\right\}(i=1 \ldots n)
$$

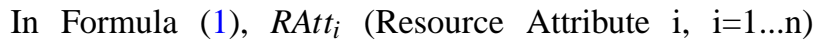
indicate a variety of properties of Internet resources, such as CPU main frequency, memory size, network bandwidth, disk apace, etc. 


\subsection{Effective and Intelligent Resource Management Model of RMMIC_ASD}

The proposed effective and intelligent resource management model of RMMIC_ASD is designed as a distributed hierarchical structure. The general infrastructure of RMMIC_ASD is shown in Fig. 1.

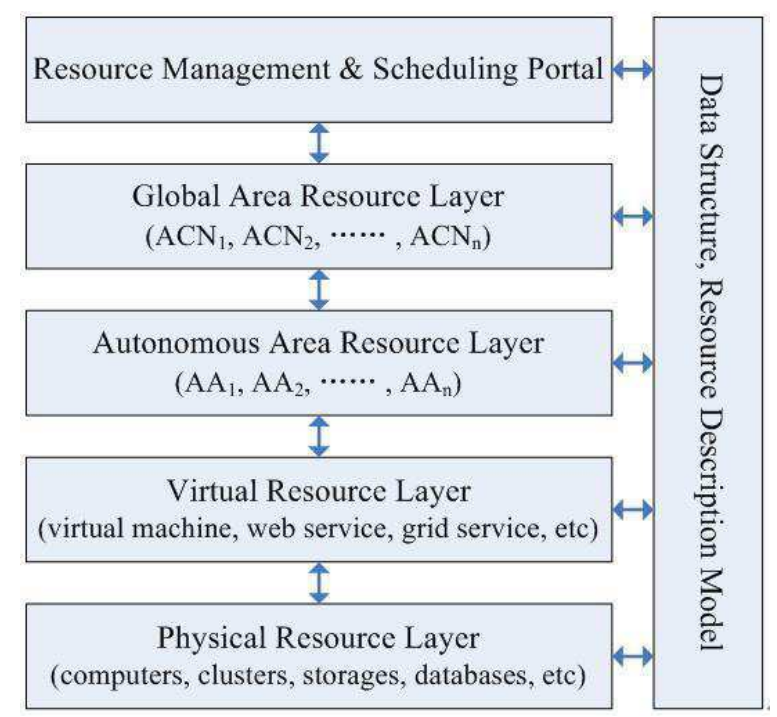

Fig. 1: General infrastructure of RMMIC_ASD (AA: Autonomous Area; ACN: Autonomous area Central Node)

As is shown in Fig. 1, the general infrastructure of RMMIC_ASD is composed of four logical layers. From bottom to top, the four layers are: the physical resource layer, the virtual service resource layer, the autonomous area resource management layer and the global area resource management layer. On the top of Fig. 1, theres a resource scheduling portal which receive users resource requests, and then the resource management model of RMMIC_ASD find and select appropriate resource to meet the users requests. The right rectangle is the public data structure and description model for the proposed model and related algorithm. To analyze the model of RMMIC_ASD more clearly, its' detailed constitution is shown in Fig. 2.

As is shown in Fig. 2, we carefully design two kinds of Agents: Node Resource Management Agent (NRMA) and Autonomous area Resource Management Agent (ARMA). Both of the two kinds of Agents are deployed in every nodes of the Internet Computing environment, and are to be activated in different occasion when needed. The agent of NRMA is designed to dynamically monitor and manage real-time resources deployed in various nodes in Internet Computing environment, while ARMA is to manage resources attached to the very autonomous area which it belongs to. The four layers of the proposed model are described in detail as following:

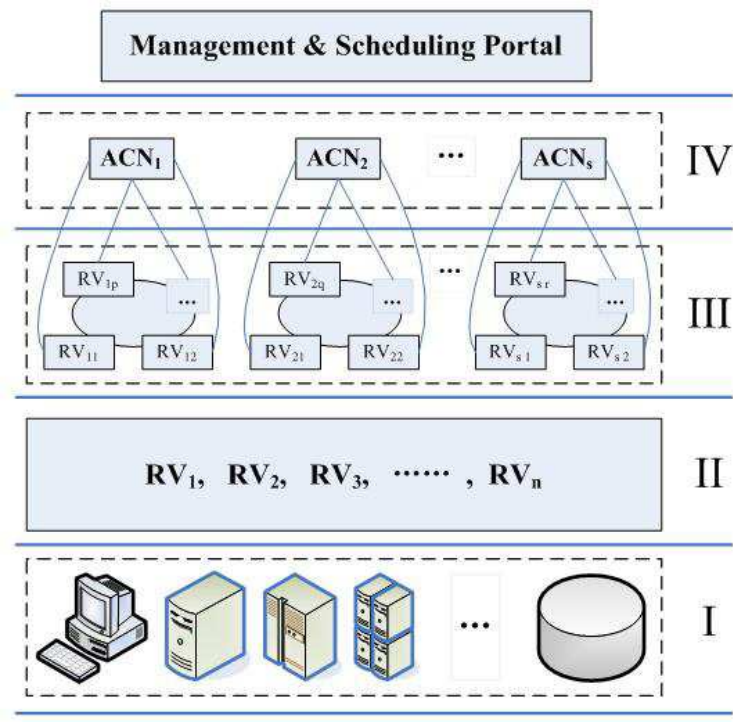

Fig. 2: Detailed constitution of RMMIC_ASD (I: The physical resource layer;)

(II: The virtual service resource layer;)

(III: The autonomous area resource management layer;)

(IV: The global area resource management layer)

(1) The physical resource layer is composed of various physical resources (such as computers, clusters, storages, databases, etc) reside in nodes of Internet Computing environment.

(2) In the virtual service resource layer, we virtualize various physical resources as Internet Service resources, such as virtual machine, web service, grid service and so on. The Internet resources are described by RVs (Resource Vectors) which is defined in formula (1).

(3) To construct the autonomous area resource management layer, we firstly deploy and activate NRMAs (Node Resource Management Agents) in every node in the Internet Computing environment; Secondly, let all NRMAs try to consult with each other, and thus to join and organize a certain number of Resource Management Autonomous Areas (RMAAs) according to SNT theory, and at the same time, every RMAA should select and define an Autonomous area Central Node (ACN) as the representation of the very RMAA; Thirdly, the Autonomous area Central Node activate the Agent of ARMA (deployed in Internet Computing node in advance), then ARMA tries to establish a chord ring and let all Internet services of the autonomous area added into the chord ring according to DHT chord algorithm.

(4) Finally, in the global area resource management layer, all Central Nodes and its ARMAs try to constitute the global resource management system 
for the global Internet Computing environment. When resource requests arrive, the resource scheduling portal firstly distribute the request to all ARMAs; Then every ARMA tries to find and select a most matched resource among its autonomous area according to the users request; Thirdly, every ARMA submit their matched resources to the scheduling portal; Fourthly, the resource scheduling portal select the most matched resource among the global Internet Computing environment according to the users request; Finally, the resource scheduling portal schedule the most matching resource to the user to accomplish his task.

\section{Effective and Intelligent Resource Management Algorithm of RMAIC_ASD}

In this section, we bring forward the resource management algorithm of RMAIC_ASD in detail.

The basic idea of the algorithm of RMAIC_ASD is as following: Firstly, by using Internet computing technologies (such as virtual machine, web service and grid computing technology and so on), let all kinds of physical resources be virtualized into Internet Services. Then we use Resource Vectors (VRs) as the representative of Inter-net Computing resources. Secondly, all Internet Computing nodes activate their prearranged resource management agent (namely NRMA), and then all NRMAs try to negotiate with each other so as to join and organize a certain number of Resource Management Autonomous Areas (RMAAs) according to SNT theory; At the same time, every RMAA should select and define an Autonomous areas Central Node $(\mathrm{ACN})$ as the representation of the very RMAA which the ACN belongs to; Then, the ACN activate ARMA (deployed in it in advance), and all ARMAs tries to establish an autonomous area Chord ring and let all Internet service resources of the RMAA added into the autonomous area Chord ring according to DHT Chord algorithm. Here divide the whole Internet Computing system into certain number of RMAAs and each RMAA has a Chord ring to organize and manage all Internet service resources of the RMAA. Finally, as the representatives of ACNs, all ARMAs consult each other so as to construct the global resource management system. In order to enhance the performance and efficiency of the whole resource management system, all ARMAs are built into a global area Chord ring according to DHT Chord algorithm.

To discuss the algorithm of RMAIC_ASD more clearly, we divide the algorithm into a main algorithm (i.e. RMAIC_ASD) and a sub one. The function of the sub algorithm is to build autonomous area resource management system. This sub algorithm should be running periodically, so that our model and algorithm can cope with dynamically changing Internet Computing environment and be ready for use in real time. In order to achieve the above goal, it should be called at three occasions: (i) When the system is initialized, the whole system should be rebuilt; (ii) When new resource request arrive and the resource management system does not ready; (iii) When any exceptions of the system occur, and the system needed to be rebuilt immediately. The sub algorithm (namely $\mathrm{Sub}_{-} \mathrm{Alg}$ ) is presented in detail as below:

(1) If (Sub_Alg should be called)

(a) Activate all NRMAs;

(b) NRMAs negotiate with each other and try to form a certain number of autonomous areas respectively according to SNT;

(c) Pick out a node with maximum connection degree and adequate computing power in every autonomous area, and make it $\mathrm{ACN}$;

(d) Activate all ARMAs in ACNs;

(e) Every ARMAs try to establish an autonomous area Chord ring and let all Internet service resources of its' area be added into the autonomous area Chord ring according to DHT;

(2) Return ARMAs and ACNs to the caller;

(3) End.

Detailed description of the main algorithm of RMAIC_ASD is as following:

(1) Accept the users resource request;

(2) If (the Internet Computing system not established or not ready)

\{

(a) Virtualize all physical resources into Internet Services and let Resource Vectors (VRs) be their representatives;

(b) Call Sub_Alg to build autonomous-area resource management system;

(c) Organize the global resource management system by using ARMAs and ACNs;

\}

(3) The resource management portal chooses an appropriate resource and schedules it to the user;

(4) End.

\section{Simulation Experiments and Results}

The simulation experiment was conducted on network simulation platform NS-2 [15] and with BRITE [16] to generate the topology for experimental network. As is known to all, NS-2 does not support agent simulation originally, so we had to extend its ability in this respect. Borrowing the ideas of Wang [17] for reference, we extend NS-2 by extending the packet structure of the NS-2, registering new protocol header and defining class 
Table 1: Conditions of simulation experiments

\begin{tabular}{c|c|c|c|c|c}
\hline No. & 1 & 2 & 3 & 4 & 5 \\
\hline$*$ & $(100,175)$ & $(150,260)$ & $(200,350)$ & $(250,450)$ & $(300,545)$ \\
\hline$* *$ & 500 & 800 & 1100 & 1400 & 1700 \\
\hline
\end{tabular}

(* Numbers of nodes and links)

(** Numbers of resource requests)

SCD Tcp which is the subclass of the TCP, so as to make NS-2 to support agent simulation. In our experiment, we use NS-2 node as Internet computing resource and two kinds of agents (i.e. NRMAs and ARMAs) are deployed in it. BRITE is an excellent universal topology generator with good representativeness, inclusiveness and interoperability, so that it can generate topology reflecting many aspects of the actual Internet topology. Hence we use BRITE to generate topology for our experiment, so as to ensure the effect of experiments.

In order to evaluate and verify the performance and efficiency of the proposed resource management model and related scheduling algorithm, we compared it with other two similar algorithms of Endurable [4] and ARM [10] under same experimental condition. The experiment scheme is shown in Table 1. As is shown in Table 1, we do simulation experiment under 5 kinds of experimental conditions. In Table 1, the line numbers of nodes and links means that we use certain number of nodes and links to generate topology for BRITE, while the line of number of resource requests is the users requests number of resources. In each condition, the simulation experiment is repeated 20 times, and the average value of the results of 20 times is taken as the final result value. Experimental results are shown in Figure 3, Figure 4 and Figure 5. Figure 3, 4 and 5 show the experimental results of makespan, average waiting time and failure ratio of resource management and scheduling algorithm respectively.

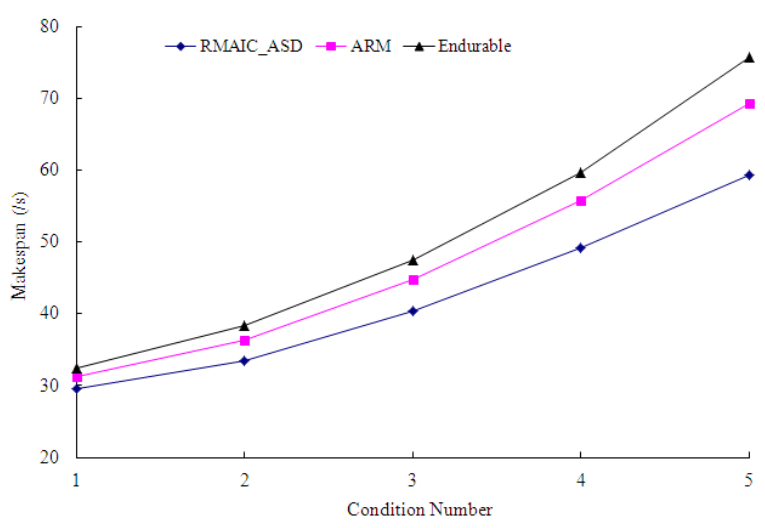

Fig. 3: Makespan of the algorithms

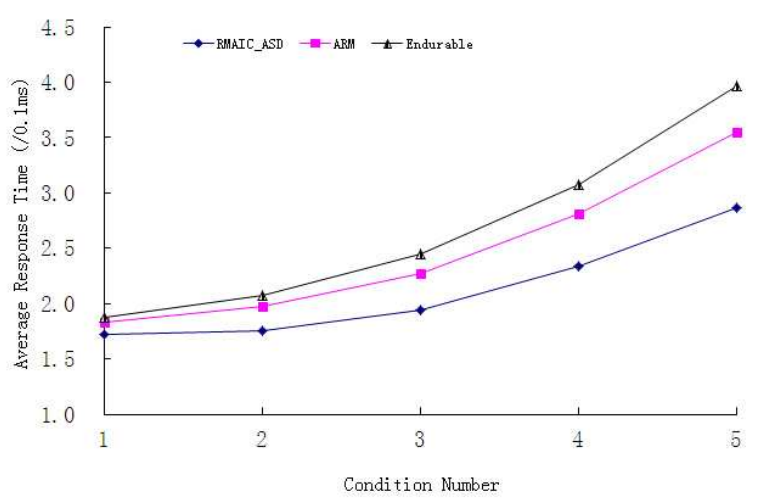

Fig. 4: Average waiting time of the algorithms

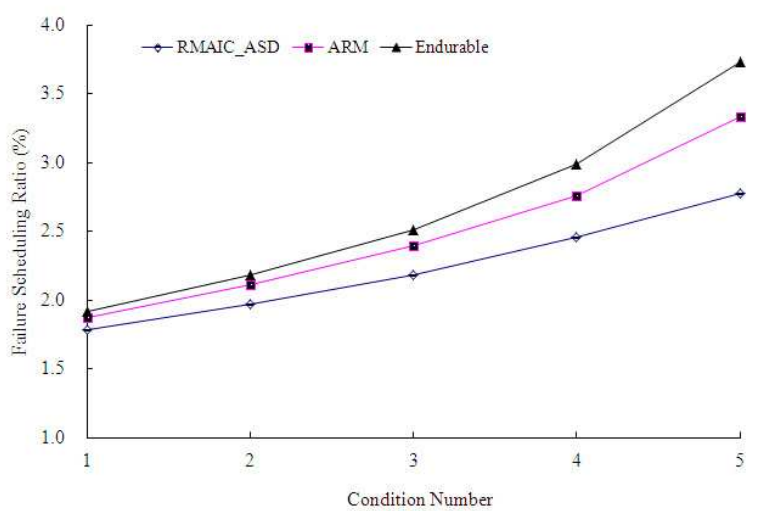

Fig. 5: Failure scheduling ratio of the algorithms

As is shown in Figure 3, 4 and 5, the proposed model and algorithm of RMMIC_ASD and RMAIC_ASD presented in this paper, works better than other two similar algorithms of Endurable and ARM. Especially when the number of resources and resource requests becoming more bigger, the algorithm of RMAIC_ASD presents more significant advantages in the respects of makespan, average waiting time and failure ratio. Based on agent technology, our model and algorithm can provide very dynamic, scalable, robust, fault-tolerant and intelligent resource management and scheduling mechanism for Internet Computing environment in real time. By using Small-world Network Theory and Distributed Hash Table to construct Internet resource management model, nodes are reachable between each other, and thus resources can be found and scheduled more quickly and efficiently. Hence, better performance and higher efficiency can be achieved in our approach. 


\section{Conclusion}

Resource management and scheduling is a key and crucial problem in Internet Computing environment. In this paper, we firstly talk about related work done in the area. Then we bring forward a novel effective and intelligent resource management model and related algorithm in internet computing environment. By using agent technology, small-world network theory and distributed hash table algorithm, the proposed model and algorithm can manage and schedule Internet Computing resources with sound intelligence, self-adaptability, robustness and high performance and efficiency. In succession, we briefly introduce the scheme and results of the simulation experiments. Experimental results show that the proposed model and algorithm are feasible and rational and works better than similar algorithms. In the future, we will continue doing more work on improving the efficiency and general performance of the model and algorithm. Furthermore, we'll try our best to make the proposed model and algorithm be applied to practical engineering projects.

\section{Acknowledgements}

This work has been co-financed by: Natural Science Foundation of Guangdong Province of China under Grant No. S2011040003843, S2011040004528 and 10351806001000000; GuangZhou Municipal High School Science Research Fund under grant No. 10A009; National Natural Science Foundation of China under Grant No. 61100150, 60903165, 61070015 and 30970191; Foundation of Comprehensive Strategic Cooperation between the Guangdong Province and the Chinese Academy of Sciences under Grant No. 2009B091300069, and Guangzhou City Council's Science \& Technology Projects Funding Scheme under Grant No. 12C42011622. We also discussed the work with the colleagues of both School of Computer Science \& Educational Software of GuangZhou University and College of Computer Science $\&$ Engineering of South China University of Technology, thanks for their self-giving helps.

\section{References}

[1] Eric M. Heien, Derrick Kondo and David P. Anderson, A Correlated Resource Model of Internet End Hosts, IEEE Transactions on Parallel and Distributed Systems, 23, 977984 (2012).

[2] Lynda Zitoune, Amel Hamdi, Veronique Veque and Hugues Mounier, Using Trajectory Tracking Control for Expressivebased SLA Applications in Internet Computing, Proc. 2009 IEEE Symposium on Computers and Communications, 1, 468-473 (2009).

[3] Jun Zhu, Zhefu Jiang and Zhen Xiao, Twinkle: A Fast Resource Provisioning Mechanism for Internet Services, Proc. IEEE INFOCOM 2011, 1, 802-810 (2011).
[4] Peng Yuxing, Wu Jiqing and Shen Rui, Distributed computing model and supporting technologies for the dynamic allocation of Internet resources, Journal of Computer Research and Development, 48, 1580-1588 (2011).

[5] Zhang Yi-Ming, Li Dong-Sheng, Lu Xi-Cheng, Scalable distri-buted resource information service for internet-based virtual computing environment, Journal of Software, 18, 1933-1942 (2007).

[6] Liu Liu, Xu Lu, Yang Dezhi, A Decentralized Resource Allocation Approach for Response-time Guarantees in Storage System, Applied Mathematics \& Information Sciences, 5, 53-60 (2011).

[7] Kesselman C., Applications of Intelligent Agent Technology to the Grid. Proc. Proceedings of IAT 2004 (IEEE/WIC/ACM 2004 International Conference on Intelligent Agent Technology), 1, xxv+569 (2004).

[8] Fufang Li, Dongqing Xie, Deyu Qi, et al., Research on Agentbased grid resource management, Computer Engineering and Applications (in Chinese), 45, 3033 (2009).

[9] Zeng Z., Shu W., Grid resource management model based on mobile agent, Computer Engineering and Applications (in Chinese), 44, 138141 (2008).

[10] Manish Mahajan, Ananthanarayanan Ramanathan and Manish Parashar, Active resource management for the differentiated services environment, International Journal of Network Management, 14, 149-165 (2004).

[11] Watts D. J. and Strogatz S. H., Collective dynamics of smallworld networks, Nature, 393, 440-442 (1998).

[12] Albert R., Barabsi A. L., Statistical mechanics of complex networks, Reviews of Modern Physics, 74, 4797 (2002).

[13] Ribe-Baumann Liz, Combining resource and location awareness in DHTs, Lecture Notes in Computer Science, 7044, 385-402 (2011).

[14] Shimano Yuta and Sato Fumiaki, Reconfiguration of Chord ring based on communication delay for lookup performance improvement, Proc. 2011 International Conference on Network-Based Information Systems (NBiS 2011), 1, 236242 (2011).

[15] NS-2 Homepage, http://www.isi.edu/nsnam/ns/index.html.

[16] BRITE (Boston university representative internet topology ge-nerator) Homepage, http://www.cs.bu.edu/brite/.

[17] Wang Yan, Wang Pei-dong and Zhang Hao, Mobile Agent si-mulation method research of extending NS2, Computer Engineering and Applications (in Chinese), 46, 64-67 (2010). 


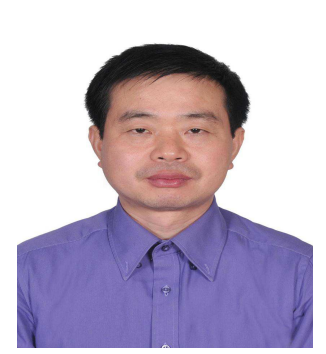

FuFang Li received the BS degree in Physics science from East China Normal University in 1991, MS degree in Computer science from Central South University in 2002, and the Ph.D. degree in Computer science from South China University of Technology in 2008. He is currently an associate professor at the School of Computer Science and Educational Software, Guangzhou University, P.R. China. He is a member of CCF (China Computer Federation), IEEE and IEEE CS. His research interests include: computer network, distributed system, grid computing, cloud computing, WSN, bioinformatics and information security.

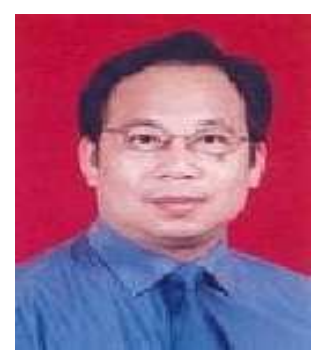

Dongqing Xie received his bachelor degree in applied mathematics from Xidian University in 1985, the M.S. degree in software engingering from Xidian University in 1988, China, and the Ph.D. degree in computer science from Hunan University in 1999, China. He is currently a professor at the School of Computer Science and Educational Software, Guangzhou University. His research interests include algorithm design and analysis, network security, graph algorithms, graph mining, applied mathematics, etc.

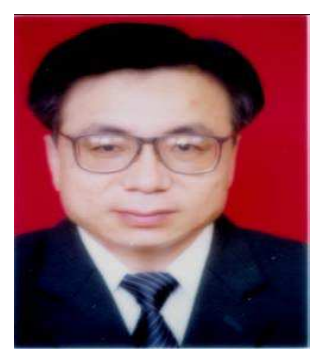

Deyu Qi received his M.S. degree in computer science and technology from National University of Defense Technology of China, and the Ph.D. degree in computer science and technology from South China University of Technology.He is a professor of School of Computer Science \& Engineering, South China University of Technology. His main research interests include: computer architecture, distributed system, information security and application, etc.

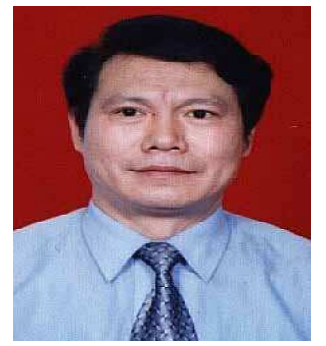

Guowen Xie received his M.S. degree from Henan Agricultural University in 1989. He is currently a professor at the School of Life Science, Guangzhou University, P.R. China. He also serves as director of the teaching and research section of botany. He is a member of Ecological Society of China and a fellow of Committee of Chinese medicine resources ecology. His main research interests include: bio-information analysis and application, genomics and application.

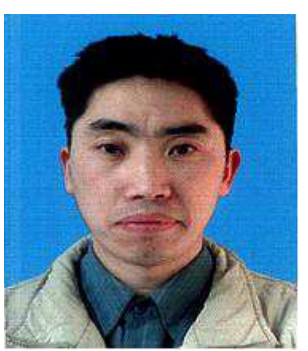

Wenbin Chen received his M.S. degree in mathematics from Institute of Software, Chinese Academy of Science in 2003, and the Ph.D. degree in computer science from North Carolina State University, U.S.A in 2010. $\mathrm{He}$ is currently an associate professor at the College of Computer Science and Educational Software, Guangzhou University. His research interests include algorithm design and analysis, bioinformatics algorithms, graph algorithms, graph mining, computational complexity, database, etc.

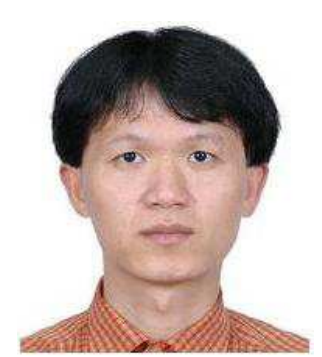

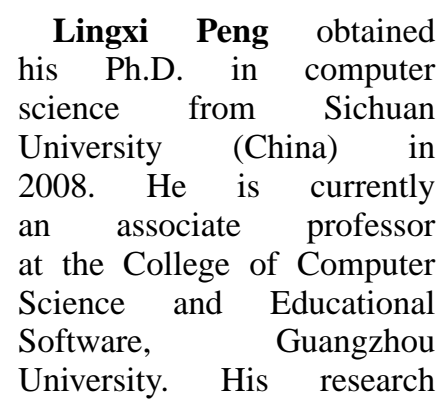
interests include artificial immune, algorithm design and analysis, bioinformatics algorithms, database, network security, graph algorithms, graph mining, computational complexity, etc.. 